\title{
Estimating Intrinsic Attenuation of a Building Using Deconvolution Interferometry and Time Reversal
}

\author{
by Conrad Newton and Roel Snieder
}

\begin{abstract}
Structural engineers have measured a building's response to strong motion from civil structures that have been instrumented with accelerometers, such as the Robert A. Millikan Library of the California Institute of Technology. The attenuation of the motion of this building has been measured using seismic interferometry techniques in the past. We use the breaking of the temporal symmetry of the wave equation by attenuation, in combination with seismic interferometry, to estimate attenuation. These estimates are made from fitting the differences in acausal and causal waveforms obtained from different deconvolution processes. We apply the method to the motion recorded at the Millikan Library and obtain estimates of intrinsic attenuation that compare well with past measurements. This technique has more precision for higher frequencies than earlier measurements that are based on seismic interferometry, and it is not dependent on radiation losses at the base of the building.
\end{abstract}

\section{Introduction}

For decades, scientists and engineers have worked to characterize building responses with the purpose of mitigating earthquake hazards and monitoring building integrity (Carder, 1936; Kuroiwa, 1967; Trifunac, 1972; Foutch, 1976; Çelebi et al., 1993; Clinton et al., 2006; Snieder and Şafak, 2006; Chopra and Naeim, 2007; Kohler and Heaton, 2007; Prieto et al., 2010). This has been done by measuring building motion, modal frequencies, intrinsic attenuation, shear velocities, and other properties. After the excitation force drives the motion of the building, intrinsic attenuation, scattering attenuation, and radiation losses dissipate the energy. Intrinsic attenuation estimates quantify the anelastic dissipation of the building's motion given by the quality factor $Q$ or the damping coefficient $\zeta$ :

$$
\zeta=\frac{1}{2 Q}
$$

Improving the measurement of intrinsic attenuation from the motion excited by complicated ground motion is the focus of our work. Advancing the measurement of attenuation, engineers can more accurately describe the motion of civil structures (Çelebi et al., 1993; Chopra and Naeim, 2007; Kohler et al., 2007), while geophysicists can produce more accurate models of the subsurface (Calvert, 2003) and diagnose the presence of fluids and the migration of these fluids in reservoirs (Bakulin et al., 2007).

Much has been learned from advanced instrumentation installed into buildings such as the Factor Building of the University of California at Los Angeles and the Millikan Library of the California Institute of Technology. These networks have produced large volumes of data for understanding wave propagation in buildings. Monitoring these types of buildings suggests that analysis be done over time to observe changes in the response of the building. Typically this analysis is done for the motion excited by earthquakes (Clinton et al., 2006; Snieder and Şafak, 2006; Kohler et al., 2007), ambient noise (Derode et al., 2003; Clinton et al., 2006; Larose et al., 2006; Prieto et al., 2010), or controlled sources (Kuroiwa, 1967; Clinton et al., 2006; Kohler and Heaton, 2007). Recently, seismic interferometry has been used for acquiring attenuation estimates (Snieder and Şafak, 2006; Kohler et al., 2007; Prieto et al., 2010).

Seismic interferometry has received much attention in the seismology community. The use of seismic interferometry has been explored for extracting Green's functions, and from this, other parameter estimations (Bakulin and Calvert, 2006; Snieder et al., 2006; Vasconcelos and Snieder, 2008; Halliday and Curtis, 2010; Wapenaar et al., 2010). Deconvolution interferometry is preferable, for reasons discussed later, for retrieving attenuation estimates. Snieder and Şafak (2006) and Kohler et al. (2007) use deconvolution interferometry for the Millikan Library and Factor Building, respectively, to acquire attenuation estimates. Our approach is similar, but we use upgoing and downgoing decomposed waves and time reversal to attain attenuation measurements.

The concept that attenuation breaks time-reversal symmetry facilitates our measurements of attenuation (Fink, 2006; Gosselet and Singh, 2007). Wave-field decomposition, in combination with deconvolution interferometry, generates acausal and causal waveforms (Snieder et al., 2006). Here, we define acausal to describe the waveforms occurring 
before time $t=0$. We compare these waveforms, and from their differences, estimate intrinsic attenuation. Because our estimate of attenuation hinges on a comparison of causal and acausal waveforms, we obtain an estimate of intrinsic attenuation because scattering attenuation is causal and invariant for time reversal. In the following, we use the term attenuation for intrinsic attenuation.

Much like the method used by Snieder and Şafak (2006), we base our estimates on a linear least-squares fit to the natural logarithm of the deconvolved waveform envelopes. We acquire accurate and precise estimates of attenuation that are frequency-dependent and compare well with previous attenuation estimates. Our method acquires frequency-dependent attenuation estimates that do not require any normal mode analysis and estimates are more precise than estimates taken from traveling waves.

The recorded shear waveforms were excited by the Yorba Linda earthquake and consist of two north-south accelerations and one east-west acceleration of the Millikan Library in 10 floors above the surface and a basement below the surface. Only one north-south acceleration dataset was used for this investigation (Fig. 1). There were some instrument coupling issues in the other two datasets as well. Building dimensions and details pertaining to the structure and instrumentation can be found in many articles, but most historical and recently notable are Kuroiwa (1967) and Clinton et al. (2006), respectively. Using only the north-south motion of the building constrains our analysis to 1 degree of freedom. The geometry of the building allows for a clamped beam model to represent the motion of the structure. The Millikan Library naturally has 3 degrees of freedom in building motion, and $3 n$ degrees of freedom if we consider a number of floors denoted by $n$ (Şafak, 1999). Our purpose is to demonstrate the application of this method, but our method can be extended to include more degrees of freedom.

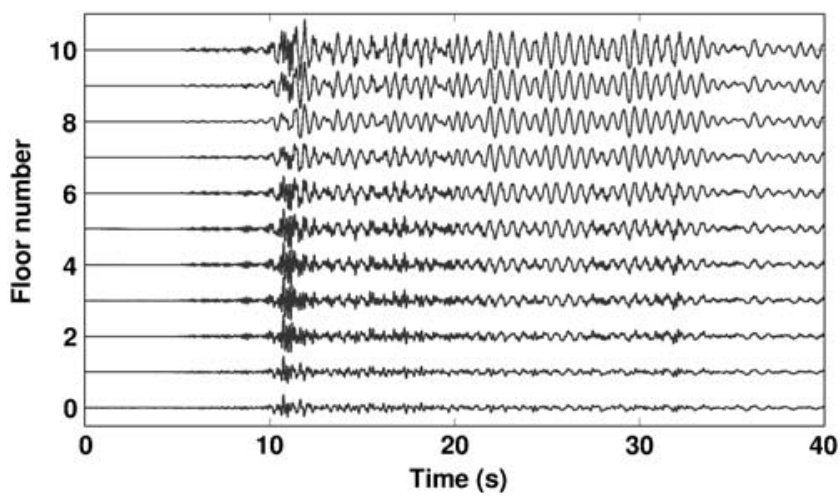

Figure 1. Yorba Linda earthquake data recorded at the Millikan Library, California Institute of Technology, Pasadena, California. The waveforms indicate accelerations in the north-south direction, and the floor numbers correspond to the location in the building where the data were recorded. Floor 0 is the basement floor.
We first discuss the basic theory behind deconvolution interferometry and time reversal. We give an example of why deconvolution interferometry is chosen, and how the deviation from time-reversal symmetry indicates the presence of attenuation and facilitates the measurement of attenuation. Next, we discuss the methodology used to achieve these results. We finish by comparing estimates of attenuation with those made from past interferometric methods of the Yorba Linda earthquake data recorded at the Millikan Library.

\section{Theory}

Seismic interferometry using deconvolution has become increasingly popular for applications in seismic imaging, parameter estimation, and passive monitoring (Curtis et al., 2006; Snieder and Şafak, 2006; Snieder et al., 2006; Kohler et al., 2007; Vasconcelos and Snieder, 2008; Prieto et al., 2010; Minato et al., 2011). Typically cross-correlation representation theorems are used to acquire the correct Green's function between receivers. Snieder (2007) shows that in the presence of dissipation, cross-correlation type seismic interferometry cannot accurately determine the attenuation response between receivers unless the medium is completely covered by sources. We first briefly explore the reasons why deconvolution seismic interferometry is preferred over crosscorrelation seismic interferometry in estimating attenuation, especially with passive seismic data.

We consider a simple 1D seismic interferometry experiment in a homogeneous dissipative medium to illustrate our decision to use deconvolution interferometry. Consider a source located at position $r_{S}$, and receivers located at $r_{A}$ and $r_{B}$ (Fig. 2). If a dissipating wave propagates away from the source and is recorded by receivers, seismic interferometry can be used to determine the response between those receivers. Seismic interferometry is a tool to measure the response between receivers, where the source position is redatumed to a known receiver location by the virtual source method (Schuster, 2009). Though the source signature of the actual source and virtual source are indeed different, the wave state obtained from seismic interferometry obeys the same wave equation as the original system (Snieder et al., 2006), and we can determine the system response to a virtual source. Examining the deconvolution and cross-correlation operations, for this example, gives insight why deconvolution is preferred for measuring attenuation. In our thought experiment, the receivers record the following frequency-domain wave fields:

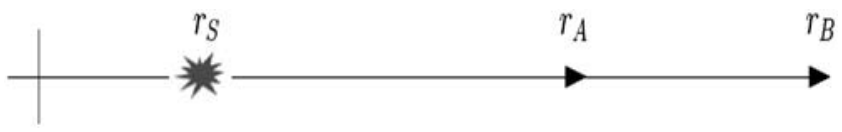

Figure 2. Definition of geometric parameters for 1D wave propagation example. 


$$
U\left(r_{A}, \omega\right)=S\left(r_{S}, \omega\right) e^{-\gamma\left(r_{A}-r_{S}\right)} e^{i k\left(r_{A}-r_{S}\right)},
$$

and

$$
U\left(r_{B}, \omega\right)=S\left(r_{S}, \omega\right) e^{-\gamma\left(r_{B}-r_{S}\right)} e^{i k\left(r_{B}-r_{S}\right)},
$$

where $S\left(r_{S}, \omega\right)$ is the source spectrum, $k$ is the wavenumber, and $\gamma$ the attenuation coefficient.

The choice of which seismic interferometric operation gives an accurate estimation of the attenuation becomes apparent from the application of each interferometric technique to the waveforms $U\left(r_{A}, \omega\right)$ and $U\left(r_{B}, \omega\right)$. Cross-correlation interferometry applied to these wave fields gives, in the frequency domain,

$$
\begin{aligned}
C C\left(r_{B}, r_{A}, \omega\right) & =U\left(r_{B}, \omega\right) U^{*}\left(r_{A}, \omega\right) \\
& =\left|S\left(r_{S}, \omega\right)\right|^{2} e^{-\gamma\left(r_{B}+r_{A}-2 r_{S}\right)} e^{i k\left(r_{B}-r_{A}\right)} .
\end{aligned}
$$

The phase is correct, but the amplitude is incorrect, because it depends on the sum of the positions $r_{B}+r_{A}$ rather than the difference $r_{B}-r_{A}$. The amplitudes estimated from cross correlation are thus incorrect for attenuation analysis. In contrast, deconvolution of the fields of equations (2) and (3) in the frequency domain gives

$$
D\left(r_{B}, r_{A}, \omega\right)=\frac{U\left(r_{B}, \omega\right)}{U\left(r_{A}, \omega\right)}=e^{-\gamma\left(r_{B}-r_{A}\right)} e^{i k\left(r_{B}-r_{A}\right)} .
$$

With deconvolution interferometry, we thus obtain the correct phase, $e^{i k\left(r_{B}-r_{A}\right)}$, and amplitude, $e^{-\gamma\left(r_{B}-r_{A}\right)}$, that account for the attenuation of the waves that propagate between the two receivers. Note that cross correlation requires the power spectrum of the source signal as well as the source location, where deconvolution interferometry is independent of the source properties. Because of these properties, we use deconvolved waveforms for our attenuation measurements. Equation (5) is potentially unstable when the reference spectrum $U\left(r_{A}, \omega\right) \rightarrow 0$. For our method, we use a stabilized deconvolution given by

$$
D\left(r_{B}, r_{A}, \omega\right)=\frac{U\left(r_{B}, \omega\right)}{U\left(r_{A}, \omega\right)} \Rightarrow \frac{U\left(r_{B}, \omega\right) U^{*}\left(r_{A}, \omega\right)}{U\left(r_{A}, \omega\right) U^{*}\left(r_{A}, \omega\right)+\epsilon},
$$

where we take $\epsilon$ to be $1 \%$ of the average power of $U\left(r_{A}, \omega\right)$.

\section{Methodology}

Attenuation of waves is expressed in the quality factor that is defined as the relative energy loss over a cycle of oscillation (Aki and Richards, 1980). Estimations of attenuation can be made by measuring the loss in the amplitudes as waves propagate between receivers. These estimations are based on the assumptions that both receivers are coupled accurately to the medium, and the amplitude picks correspond to the same seismic event. Previous studies of Snieder and Şafak (2006) measured attenuation with the Millikan
Library data using interferometry to reduce the imprint of a variable receiver coupling. Our method estimates attenuation from individual recordings deconvolved with a common signal, and estimates can be averaged over the array of recordings to reduce and estimate the error. Deconvolution interferometry with a reference signal decomposed into upgoing and downgoing waves generates wave states with an impulsive upgoing and downgoing wave, respectively, at the base of the building (Snieder et al., 2006). The upgoing and downgoing waveforms of an individual receiver can be compared to give measurements of attenuation.

We separate the wave field at the base of the building into upgoing $\left(u_{+}\right)$and downgoing $\left(u_{-}\right)$waves using the following decomposition (Robinson, 1999):

$$
\frac{\partial u_{+}}{\partial t}=\frac{1}{2}\left(\frac{\partial u}{\partial t}-c \frac{\partial u}{\partial z}\right)
$$

and

$$
\frac{\partial u_{-}}{\partial t}=\frac{1}{2}\left(\frac{\partial u}{\partial t}+c \frac{\partial u}{\partial z}\right)
$$

The $z$-derivative follows from the difference of the motion recorded in the basement and on the first floor. We use the value $c=322 \mathrm{~m} / \mathrm{s}$ as determined by Snieder and Şafak (2006).

Deconvolving the waveforms with their upgoing and downgoing waves separates the signal into causal and acausal waves (Snieder et al., 2006). The causal and acausal waveforms would be symmetric in time if attenuation were not present. We measure the attenuation from the differences in the causal and time-reversed acausal waveforms of each floor. The procedure begins by directionally separating the wave fields in the reference floor using equations (7) and (8). We choose the basement floor recording as our reference signal and separate the signal into upgoing and downgoing waves. We estimate the $z$-derivative from the differences in the motion recorded in the basement and at the first floor. This generates waveforms that are either causal or acausal in the floors above the basement. This procedure simulates a pure upgoing or downgoing impulsive virtual source in the basement at $t=0$.

Using only the upgoing waves in the reference signal, our interferometry method extracts the building response from an upgoing impulsive source in the basement of the building at $t=0$. The deconvolutions of all the floors, in the frequency domain, are ratios of the full wave-field spectra of an individual floor and the upgoing wave spectra of the reference floor. We apply this type of deconvolution to all the floors to get 11 deconvolved waveforms (Fig. 3a). Deconvolution, with the upgoing waves in the reference floor, compresses all the upgoing waves in the basement into one upgoing virtual impulse injected at $t=0$. The response of the building to this virtual source is nonzero for times $t>0$. 

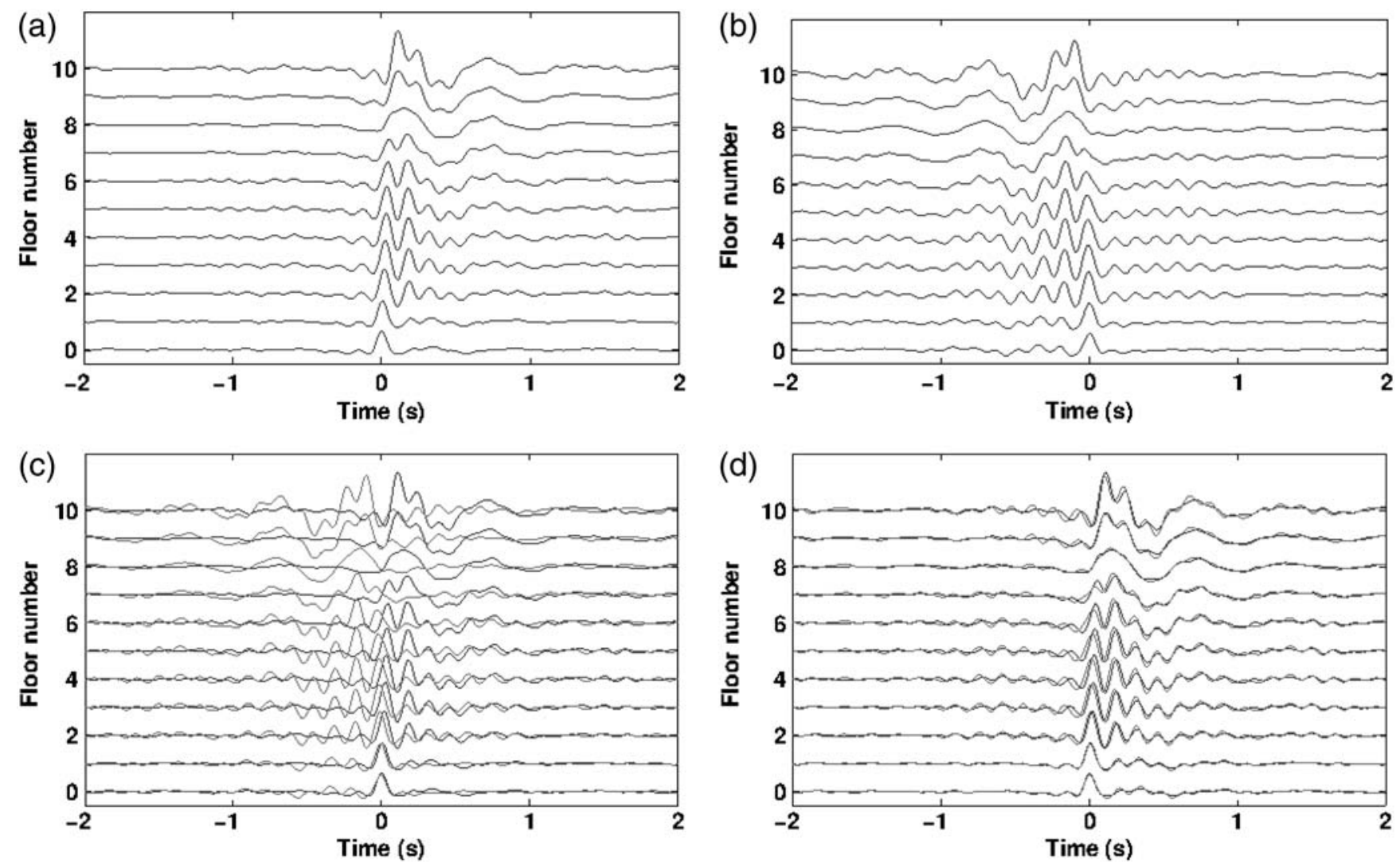

Figure 3. Waveforms deconvolved with decomposed waves and their superpositions both before and after time reversal. (a) Waveforms obtained by deconvolving the waves at every floor with the upgoing wave in the basement, (b) with the downgoing wave in the basement, (c) superposition of causal and acausal waveforms of (a) and (b), and (d) superposition after time reversal of acausal waveforms.

The use of the upgoing waves for deconvolution generates causal waveforms because the building only oscillates after the upgoing wave enters the building. In a similar manner, we also deconvolve the full waveforms of each individual floor with the downgoing waveform from the basement signal. This downgoing wave is the result of upgoing waves entering the building at earlier times (i.e., $t<0$ ). Therefore, this procedure generates acausal waveforms (Fig. 3b). Collapsing all the downgoing waves in the basement to one downward impulse requires energy to be present in the building before time $t=0$, which corresponds to the acausal waveforms seen in Figure $3 b$.

A superposition of the causal and acausal waveforms of Figure 3a,b as shown in Figure 3c, reveal a quasi-symmetry of the waveforms around $t=0$. Time reversal breaks down under certain conditions, such as rotation, flow, and intrinsic attenuation (Fink, 2006). The intrinsic attenuation in the building has broken the time-reversal symmetry of the waveforms in Figure 3c. This is apparent from Figure 3d, where the acausal waveforms generated from the deconvolution using downgoing waves of the reference floor, have been time reversed. This plot shows the superposition of the timereversed acausal waveforms and causal waveforms. Note that the amplitudes do not match, and from this difference in amplitudes we measure attenuation.
We band-pass filter the deconvolved signals using Butterworth filters of the third and second order to the respective dominant frequency bands $0.2-3.0 \mathrm{~Hz}$ and $5.0-7.8 \mathrm{~Hz}$ of the power spectra shown in Figure 4. This allows us to retrieve constant $Q$-values within each frequency band chosen for analysis, thereby yielding a frequency-dependent $Q$ in a discrete sense. After band-pass filtering our deconvolved signals using the frequency bands of Figure 4, we compute the envelopes of all the waveforms. To demonstrate the

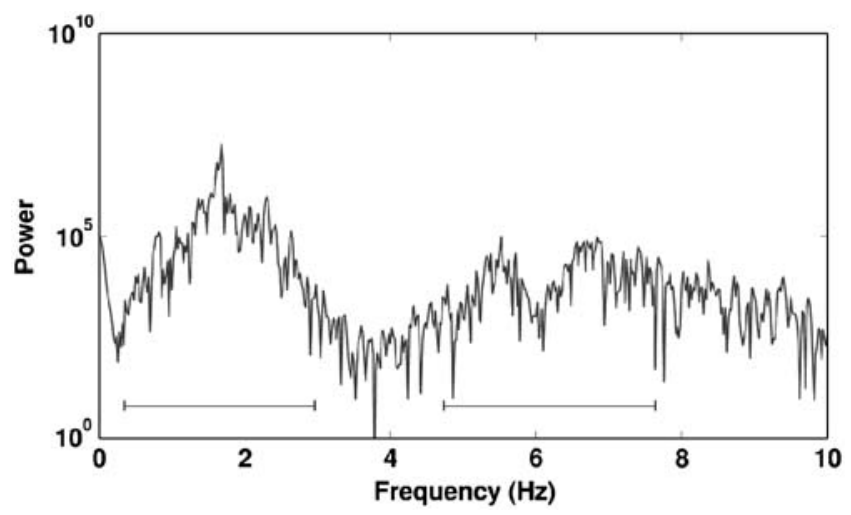

Figure 4. The root mean square power spectra of floor 7 with horizontal bars indicating the frequency bands used for band-pass filtering. 


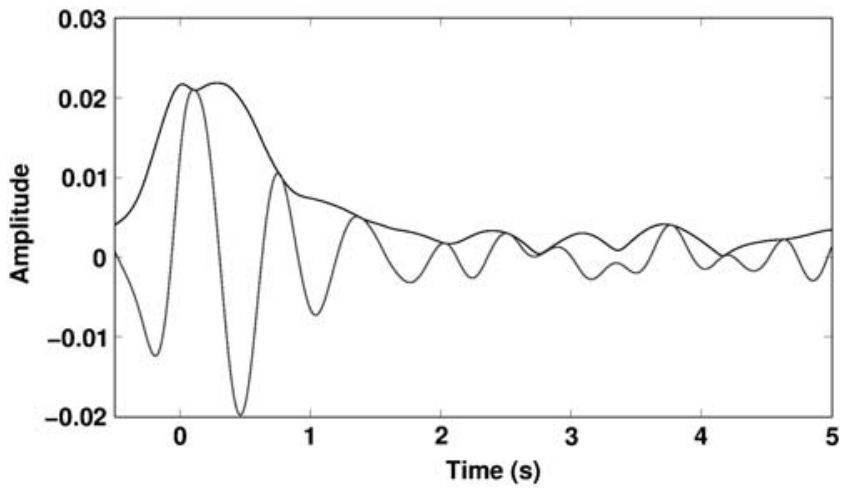

Figure 5. Signal from floor 4 deconvolved with upgoing waves after low band-pass filtering and envelope of the corresponding signal.

estimation of attenuation, we discuss the procedure for the low-frequency band-passed data in detail.

The two curves depicted in Figure 5, are a deconvolved waveform from the upgoing wave of floor 4 and the envelope corresponding to this waveform. This deconvolved waveform is generated from the spectra of the fourth floor and the

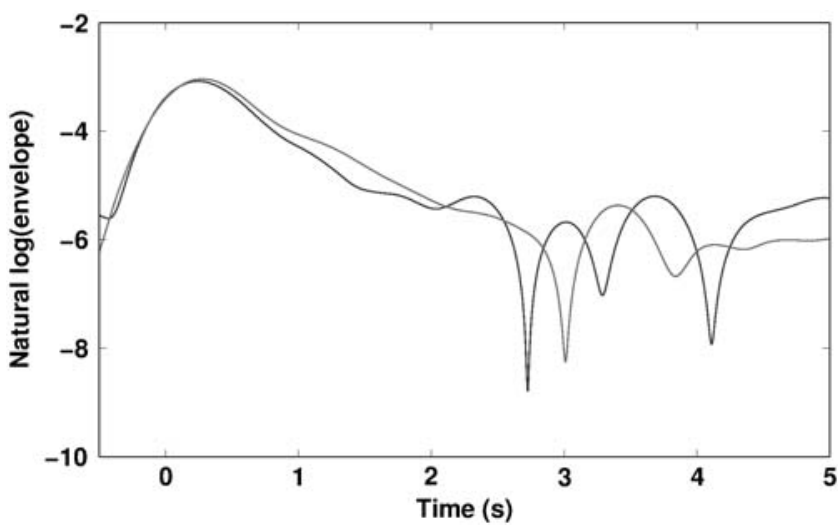

Figure 6. The natural log of envelopes from upgoing and downgoing deconvolved waveforms after low frequency $0.2-3.0 \mathrm{~Hz}$ band-pass filtering.

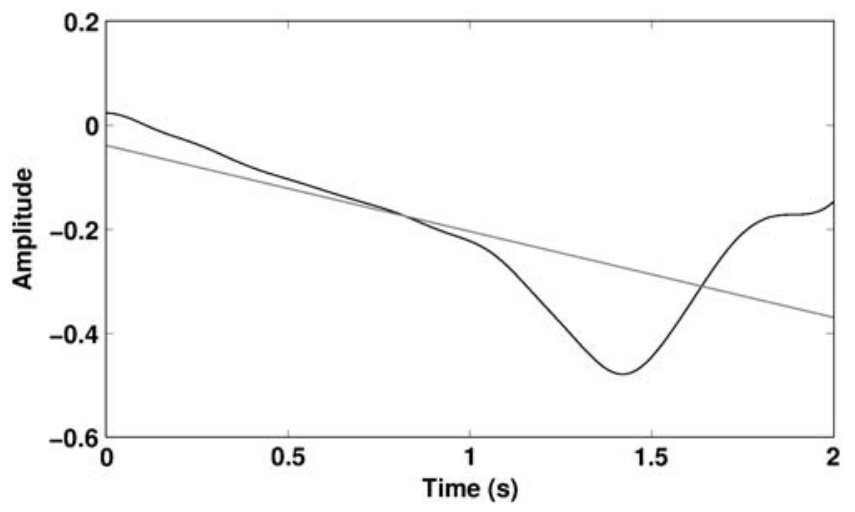

Figure 7. The difference of the curves in Figure 6 and linear fit. upgoing wave at the basement. We then apply the lowfrequency Butterworth band-pass filter of order 3 to this deconvolved waveform. The envelope is acquired from the modulus of the analytic signal using the Hilbert transform. We next take the natural logarithm of this waveform (Fig. 6). The second curve is the natural logarithm of the envelope of the wave from the fourth floor obtained from deconvolution using the downgoing wave and time reversed.

The first observation is that these two curves are not the same; this difference is due to attenuation. The second observation is that these curves decay almost linearly for the first $2 \mathrm{~s}$. During this time duration, the difference of these natural logarithms is also linear with respect to time, and a constant $Q$-value model corresponds to the slope of the difference of these curves. This model is set up by examining the envelopes of the deconvolved signals of an individual floor. We define the envelopes of the deconvolved signals to be

$$
d_{+}(t)=A_{+} e^{-m t}, \quad d_{-}(-t)=A_{-} e^{+m t} .
$$

Taking the natural logarithm of the ratio of $d_{+}$to $d_{-}$yields an equation suited for a linear regression where the slope parameter solves for the attenuation coefficient $m$ in a leastsquares sense:

$$
\ln \left|\frac{d_{+}}{d_{-}}\right|=-2 m t
$$

This model does make the assumption that the initial amplitudes are approximately equal, such that $A_{+} \approx A_{-}$. Figure 7 shows the difference of the curves of Figure 6 , for the initial $2 \mathrm{~s}$, and the linear least-squares fit. This procedure is repeated for all the floors, excluding floor 8 because of receiver coupling issues, to generate estimates of the attenuation coefficient.

We also use this procedure for the higher frequency band-pass filtered data of 5.0-7.8 Hz. In this case, the linear trend of the difference of natural logarithms of the envelopes only has a 1-s duration. The higher frequency content of the signal is expected to lead to a more rapid decay of the envelope than of the low-frequency content. Figure 8 shows that noise dominates the signal after 1-s duration because the envelope stabilizes to a near-constant value after that time. Therefore, we do our fitting within the first second, and this fitting of the difference of the curves of Figure 8 can be seen in Figure 9.

To estimate the error in our measurement of $\zeta$ due to errors in the slope of the fitting curve and the width of the employed frequency band, we use the following equations. If we write the attenuation coefficient as

$$
m=\bar{\omega} \zeta
$$

where $\bar{\omega}$ is the weighted mean of the angular frequency for a given frequency band, and the attenuation coefficient $m$ is 


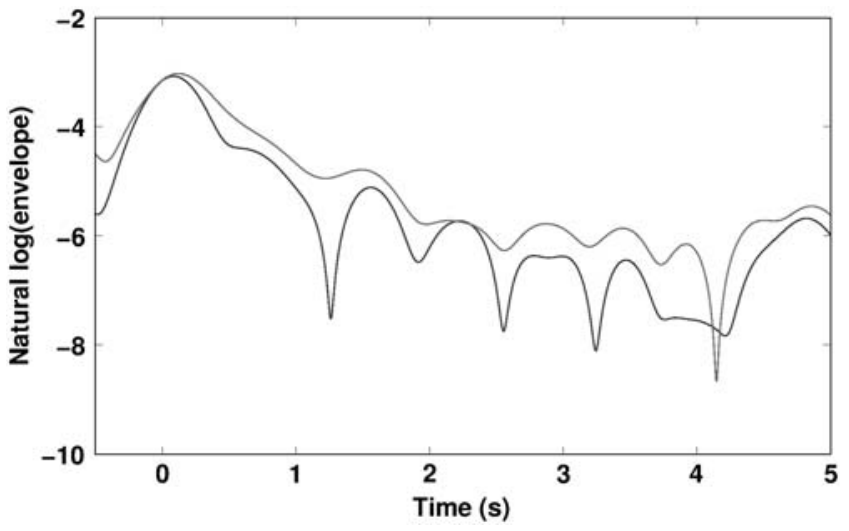

Figure 8. The natural log of envelopes from upgoing and downgoing deconvloved waveforms after high-frequency $5.0-7.8 \mathrm{~Hz}$ band-pass filtering.

given by the slope from our fitting curve, we estimate the error for the $i$-th floor using

$$
\sigma_{\zeta, i}=\sqrt{\frac{m_{i}^{2}}{\bar{w}^{2}}\left(\frac{\sigma_{m, i}^{2}}{m_{i}^{2}}+\frac{\sigma_{\omega}^{2}}{\bar{\omega}^{2}}\right)},
$$

where $\sigma_{\omega}$ is our standard deviation of $\bar{\omega}$ given by

$$
\bar{\omega}=\frac{\int_{\Omega} \omega P(\omega) d \omega}{\int_{\Omega} P(\omega) d \omega},
$$

and

$$
\sigma_{\omega}^{2}=\frac{\int_{\Omega}(\omega-\bar{\omega})^{2} P(\omega) d \omega}{\int_{\Omega} P(\omega) d \omega}
$$

where $P(\omega)$ is the power spectrum within the employed frequency band $\Omega$. Our standard deviation is $\sigma_{m, i}$ of the attenuation coefficient of the $i$-th floor from estimates of the discrepancy of the data from the least-squares linear fit (Bevington and Robinson, 2003). This procedure for $\sigma_{\zeta, i}$ is repeated and averaged for all the floors except the eighth floor. Equation (12) is based on the assumption that the frequency and slope are independent measurements, and

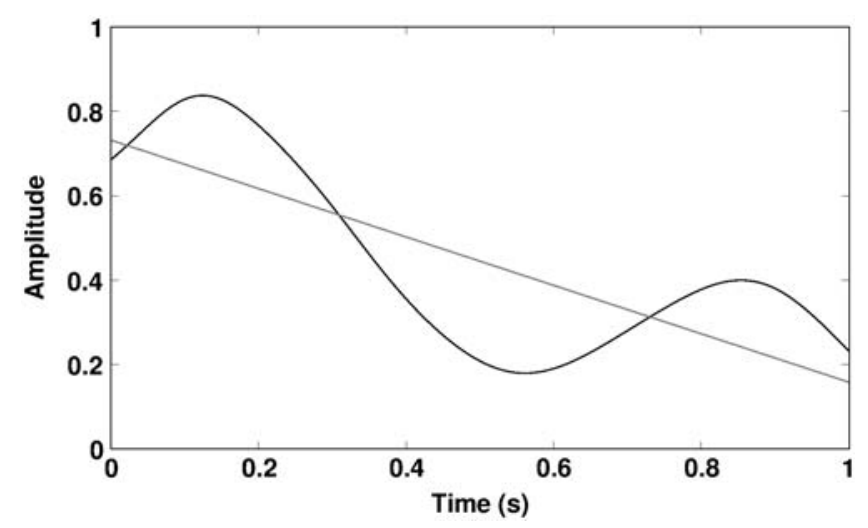

Figure 9. The difference of the curves in Figure 8 and linear fit.
Table 1

Damping Coefficients

\begin{tabular}{lccc}
\hline Method & $\zeta$ & $\sigma_{\zeta}$ & Frequency Mode \\
\hline $\mathrm{TR}^{*}$ & $1.14 \%$ & $0.50 \%$ & Fundamental \\
$\mathrm{NM}^{\dagger}$ & $1.15 \%$ & $0.41 \%$ & Fundamental \\
Newton and Snieder & $1.57 \%$ & $\mathrm{NA}$ & Fundamental \\
$\quad$ (half power) & & & \\
Bradford et al., 2004 & $2.39 \%$ & $\mathrm{NA}$ & Fundamental \\
Clinton et al., 2006 & $1.63 \%$ & $\mathrm{NA}$ & Fundamental \\
Kuroiwa, 1967 (half power) & $1.54 \%$ & $\mathrm{NA}$ & Fundamental \\
Kuroiwa, 1967 (mode shape) & $1.47 \%$ & $\mathrm{NA}$ & Fundamental \\
Kuroiwa, 1967 (Hudson's) & $1.74 \%$ & $\mathrm{NA}$ & Fundamental \\
TR* & $1.74 \%$ & $0.39 \%$ & First overtone \\
TW & $1.58 \%$ & $1.36 \%$ & First overtone \\
\hline
\end{tabular}

*TR, time reversal.

${ }^{\dagger} \mathrm{NM}$, normal mode.

†TW, traveling wave.

therefore that their covariance vanishes. The attenuation estimates with their errors are presented in Table 1.

\section{Relation to Previous Work}

The motion of the Millikan Library has been used before to estimate intrinsic attenuation using deconvolution interferometry (Snieder and Şafak, 2006), and we compare our results with these past measurements. Snieder and Şafak (2006) developed two techniques using deconvolution interferometry. A technique that measures attenuation from the normal mode oscillation of the building and another that measures attenuation from higher frequency traveling waves.

The technique employing the normal mode oscillations of the building uses the basement floor signal as the reference signal for the deconvolution defined by equation (6). This technique, however, does not decompose the wave field into up and downgoing waves. Using the full spectra of the reference signal, we generate the deconvolution waveforms in Figure 10. In this figure, the motion at the basement floor is compressed to a band-limited spike at $t=0$. For $t>0$, the figure shows the response of the building to the impulsive

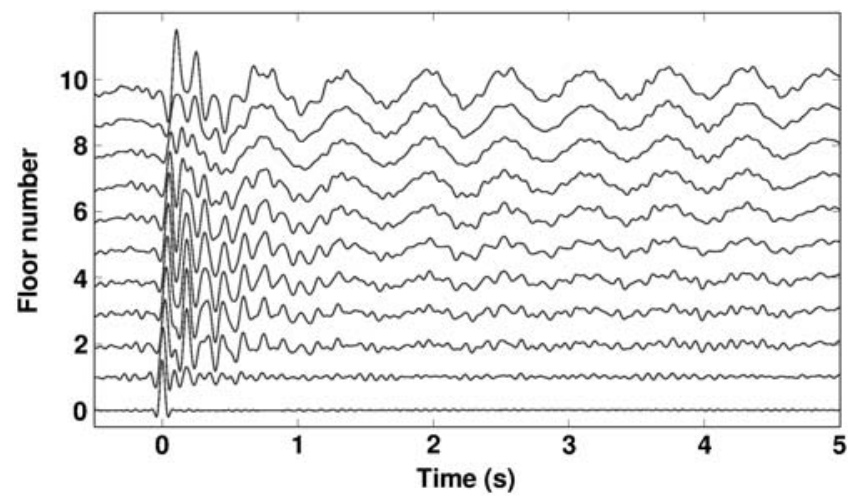

Figure 10. The motion of the building in Figure 1 after deconvolution with the motion recorded in the basement. 
excitation. This response is dominated by the fundamental mode of the building with a period of about $0.6 \mathrm{~s}$.

We first band-pass filter the waveforms of Figure 10 between $0.2-3.0 \mathrm{~Hz}$, using the low-frequency range indicated by the left horizontal bar in Figure 4. A linear curve is fit to the natural logarithm of the envelopes of the deconvolved waveforms similar to our time-reversal method. Figure 11 shows the natural logarithm of the envelope of the signals in Figure 10 in solid lines and the least-squares fits in dashed lines. The slope of the least-squares fit is proportional to the attenuation coefficient. Table 1 shows the average damping estimate corresponding to the normal mode measurement indicated by NM for this method. An attempt to measure the damping with this method at the higher frequency band yielded poor results. This is due to low amplitude in the power spectrum of the frequency band of 5.0-7.8 Hz.

The next deconvolution interferometry technique developed by Snieder and Şafak (2006) uses higher frequency traveling waves. Snieder and Şafak (2006) make their attenuation measurements using the top floor signal as the reference signal for the deconvolution. Note that there is no decomposition of the wave fields at the reference floor for this traveling wave procedure. The deconvolved waveforms in Figure 12 use the full spectra of each signal. In Figure 12 a

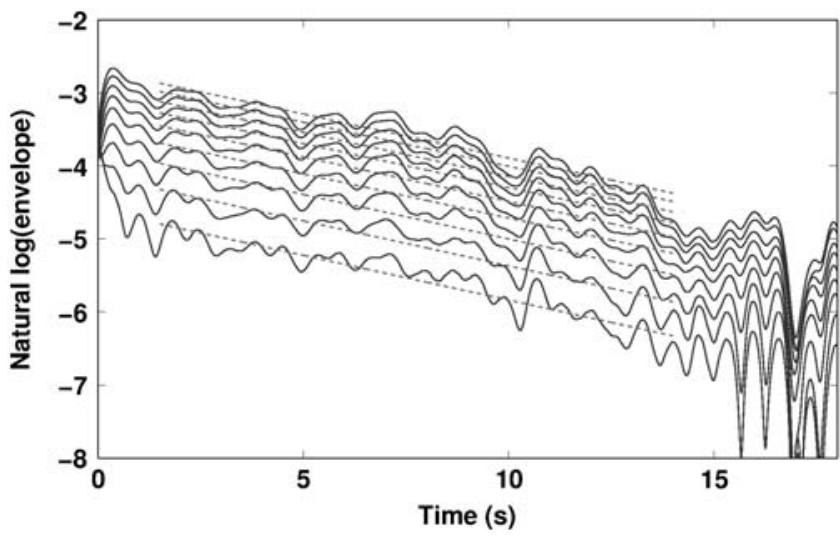

Figure 11. Natural log of envelopes from signals in Figure 10 and linear fit.

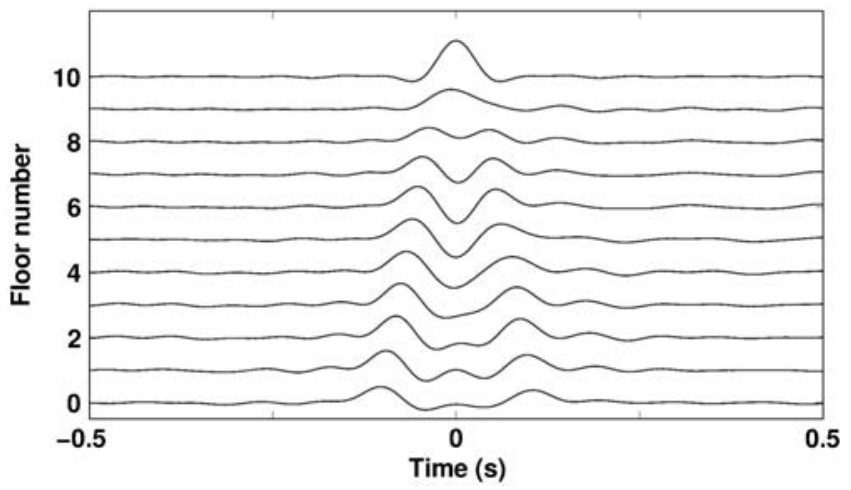

Figure 12. The motion of the building in Figure 1 after deconvolution with the motion at the top floor. traveling wave moves up and then down the building. Knowing the shear velocity of the building, the ratio of the amplitudes of the upgoing and downgoing waves, the distance traveled from each receiver to the top of the building and back down to the receiver, and the travel times, one can estimate the attenuation. Table 1 displays the results of these measurements marked TW for the traveling wave technique. Because this estimate depends only on the amplitude ratio of the upgoing and downgoing waves at each floor, this estimate is not affected by variations in receiver coupling. The observation that the signals are quiescent after the traveling wave has moved up and down through the building in Figure 12 also suggests there is little scattering caused by the individual floors.

\section{Discussion}

Table 1 shows that our method of using deconvolution interferometry with time reversal gives estimates of attenuation that compare well with values found previously using seismic interferometry and classical methods. The new method we propose has several benefits compared with the past methods. Our method recovers attenuation estimates in the normal mode and the first overtone. This makes the new proposed method more robust than the past interferometric methods because of the proposed method's ability to perform at higher frequencies. Classical methods, such as the halfpower method, lose their robustness in higher overtones because of uncertainty in the half-power amplitude picks on either side of the peak frequency for a given overtone.

Our proposed method removes the radiation damping from the global attenuation estimate, leaving only internal mechanisms for energy loss such as scattering attenuation, constant Coulomb internal friction, and intrinsic material attenuation. Our method shares the ability to separate the radiation damping with past seismic interferometric methods. Table 1 gives estimates that employ classical modal analysis that are available in the literature (Kuroiwa, 1967; Bradford et al., 2004; Clinton et al., 2006). These methods recover global damping values of the soil-structure system. These values include radiation damping that occurs at the soilstructure interface. Our method using deconvolution interferometry with time reversal removes the radiation damping by allowing the energy to leave the system. For instance, the upgoing deconvolution used in our proposed method simulates one upgoing wave injected into the building in the basement at time $t=0$. The wave moves up and then down through the building and continues down. In essence, there is a reflection coefficient of 0 in the basement. This leaves only internal mechanisms of attenuation in our deconvolved signals from which we measure. Figure 12, of the traveling wave experiment, indicates that there may be little contribution from scattering attenuation. Combining classical methods with our proposed method could refine our knowledge of civil structure behavior and improve earthquake modeling. 
The classical modal analysis done by Kuroiwa (1967) includes techniques such as half-power, mode shape, and Hudson's method. The half-power method attenuation estimate is approximately the width between the peak amplitude where the amplitude value is $\sqrt{2} / 2$ times the peak amplitude. Kuroiwa (1967) uses normalized deflections, force applied, and accelerations at individual floors to derive an estimate of attenuation within the normal mode frequency. Kuroiwa (1967) also uses the Hudson method to measure attenuation using the peak amplitude and amplitude where the response curve is horizontal in each signal. For more details on these methods of classical modal analysis, see Kuroiwa (1967).

Our method is built upon a linear mathematical framework that may lead to limitations in this method and require an attenuation model representation beyond the constant $Q$ description. Our method is described by a linear elastic behavior of the medium, and strong shaking may invalidate the assumption of linearity. Structures proximal to epicenters of large earthquakes will have strong ground motion excitation that can generate nonlinear effects because the mechanical properties of the building may depend on excitation levels (Clinton et al., 2006). In this dataset for the Millikan Library we did not observe many internal reflections and consequently little scattering attenuation. Datasets with stronger scattering attenuation may introduce complications to this method's ability to accurately measure intrinsic attenuation.

In the future, this method should be tested on more data to further understand the limitations of this method. Elements of nonlinearity and other sources of attenuation could pose problems for this method. Other civil structures with different geometries such as other building designs, bridges, and down-hole arrays could also benefit from this method of attenuation investigation. An extension to include higher degrees of freedom would benefit this method to perform in more complex structures. This new method of deconvolution interferometry with time reversal has proved to be a viable method for this dataset and should continue to be explored for further advanced applications.

\section{Conclusion}

We have shown, using data recorded in the Millikan Library, that deconvolution interferometry with time reversal is an effective method to measure attenuation in civil structures. This method extracts estimates of intrinsic attenuation from the breaking of time-reversal symmetry. By timereversing the acausal waveforms, we estimate intrinsic attenuation using a fitting procedure that is similar to past methods using normal mode oscillations. By comparing the estimates of our time-reversal method with that of past seismic interferometry methods, we have shown that our results compare well with the past methods of Snieder and Şafak (2006). Additionally, the time-reversal method has higher precision than the traveling wave method and not constrained to measuring only the fundamental mode.

\section{Data and Resources}

The data are recorded and made available through the National Strong Motion Project Data Sets of the U.S. Geological Survey. The data for the Yorba Linda earthquake can be accessed through http://nsmp.wr.usgs.gov/data_sets/ 20020903_1.html\#Downloads (last accessed March 2012). Bradford et al.'s (2004) results of Millikan Library forced vibration testing can be found at http://resolver.caltech.edu/ CaltechEERL:EERL-2004-03 (last accessed March 2012).

\section{Acknowledgments}

This work was supported by the Consortium Project on Seismic Inverse Methods for Complex Structures at Center for Wave Phenomena (CWP). Thank you to the anonymous reviewers for their valuable suggestions that helped improve this article.

\section{References}

Aki, K., and P. G. Richards (1980). Quantitative Seismology: Theory and methods, W. H. Freeman, San Francisco, California, 161-187.

Bakulin, A., and R. Calvert (2006). The virtual source method: Theory and case study, Geophysics 71, SI139-SI150.

Bakulin, A., A. Mateeva, K. Mehta, P. Jorgensen, J. Ferrandis, I. S. Herhold, and J. Lopez (2007). Virtual source applications to imaging and reservoir monitoring, The Leading Edge 26, 732-740.

Bevington, P., and D. Robinson (2003). Error Analysis, in Data Reduction and Error Analysis for the Physical Sciences, McGraw-Hill, New York, 36-46.

Bradford, S. C., J. F. Clinton, J. Favela, and T. H. Heaton (2004). Results of Millikan Library forced vibration testing, Tech. Rept., California Institute of Technology, Pasadena, California..

Calvert, R. W. (2003). Seismic imaging a subsurface formation, U.S. Patent 20030076740.

Carder, D. S. (1936). Observed vibrations of buildings, Bull. Seismol. Soc. Am. 26, 245-277.

Çelebi, M., L. T. Phan, and R. D. Marshall (1993). Dynamic characteristics of five tall buildings during strong- and low-amplitude motions, The Structural Design of Tall Buildings 2, 1-15.

Chopra, A. K., and F. Naeim (2007). Dynamics of structures: Theory and applications to earthquake engineering, Third Ed., Earthquake Engineering Research Institute23, 409-414.

Clinton, J. F., S. C. Bradford, T. H. Heaton, and J. Favela (2006). The observed wander of the natural frequencies in a structure, Bull. Seismol. Soc. Am. 96, 237-257.

Curtis, A., P. Gerstoft, H. Sato, R. Snieder, and K. Wapenaar (2006). Seismic interferometry-Turning noise into signal, The Leading Edge 25, 1082-1092.

Derode, A., E. Larose, M. Campillo, and M. Fink (2003). How to estimate the Green's function of a heterogeneous medium between two passive sensors? Application to acoustic waves, Appl. Phys. Lett. 83, 3054-3056.

Fink, M. (2006). Time-reversal acoustics in complex environments, Geophysics 71, SI151-SI164.

Foutch, D. A. (1976). A study of the vibrational characteristics of two multistory buildings, Ph.D. Thesis, California Institute of Technology, Earthquake Engineering Research Laboratory, Pasadena, California.

Gosselet, A., and S. C. Singh (2007). Using symmetry breaking in timereversal mirror for attenuation determination, SEG Annual Meeting Expanded Technical Program Abstracts 26, 1639-1643.

Halliday, D., and A. Curtis (2010). An interferometric theory of sourcereceiver scattering and imaging, Geophysics 75, SA95-SA103. 
Kohler, M. D., and T. H. Heaton (2007). A time-reversed reciprocal method for detecting high-frequency events in civil structures, American Geophysical Union Fall Meeting Abstracts, B1265-1272.

Kohler, M. D., T. H. Heaton, and S. C. Bradford (2007). Propagating waves in the steel, moment-frame Factor Building recorded during earthquakes, Bull. Seismol. Soc. Am. 97, 1334-1345.

Kuroiwa, J. (1967). Vibration tests of a multistory building, Ph.D. Thesis, California Institute of Technology, Earthquake Engineering Research Laboratory, Pasadena, California.

Larose, E., L. Margerin, A. Derode, B. van Tiggelen, M. Campillo, N. Shapiro, A. Paul, L. Stehly, and M. Tanter (2006). Correlation of random wavefields: An interdisciplinary review, Geophysics $\mathbf{7 1}$, SI11-SI21.

Minato, S., T. Matsuoka, T. Tsuji, D. Draganov, J. Hunziker, and K. Wapenaar (2011). Seismic interferometry using multidimensional deconvolution and cross correlation for crosswell seismic reflection data without borehole sources, Geophysics 76, SA19-SA34.

Prieto, G. A., J. F. Lawrence, A. I. Chung, and M. D. Kohler (2010). Impulse response of civil structures from ambient noise analysis, Bull. Seismol. Soc. Am. 100, 2322-2328.

Robinson, E. (1999). Seismic inversion and deconvolution, Chapter 1, in Handbook of Geophysical Exploration, 4B: Pergamon, Oxford, UK, $1-32$.

Şafak, E. (1999). Wave-propagation formulation of seismic response of multistory buildings, J. Struct. Eng. 125, 426-437.

Schuster, G. T. (2009). Seismic interferometry: Cambridge University Press, Cambridge, 1-15, 30-50.
Snieder, R. (2007). Extracting the Green's function of attenuating heterogeneous acoustic media from uncorrelated waves, J. Acoust. Soc. Am. 121, 2637-2643.

Snieder, R., and E. Şafak (2006). Extracting the building response using seismic interferometry: Theory and application to the Millikan Library in Pasadena, California, Bull. Seismol. Soc. Am. 96, 586-598.

Snieder, R., J. Sheiman, and R. Calvert (2006). Equivalence of the virtualsource method and wave-field deconvolution in seismic interferometry, Phys. Rev. E 73, 066620.

Trifunac, M. D. (1972). Comparisons between ambient and forced vibration experiments, Earthquake Eng. Struct. Dyn. 1, 133-150.

Vasconcelos, I., and R. Snieder (2008). Interferometry by deconvolution: Part 1-theory for acoustic waves and numerical examples, Geophysics 73, S115-S128.

Wapenaar, K., D. Draganov, R. Snieder, X. Campman, and A. Verdel (2010). Tutorial on seismic interferometry: Part 1-Basic principles and applications, Geophysics 75, 75A195-75A209.

Center for Wave Phenomena

Department of Geophysics

Colorado School of Mines

1500 Illinois Street

Golden, Colorado 80401

cnewton@mines.edu

rsnieder@mines.edu

Manuscript received 17 November 2011 